\title{
Study on Intermittent WLAN Consisting of Heterogeneous Multi-radio Devices
}

\author{
Xue Yang, Jing Zhu and Xingang Guo
}

\author{
xue.yang, jing.z.zhu, xingang.guo @ intel.com \\ Communication Technology Lab, Intel Corporation, Hillsboro, OR, 97124, USA
}

\begin{abstract}
It is envisioned that multiple radios may be integrated into a single portable device in the near future. Such multi-radio devices may participate in multiple networks at the same time. In this paper, we consider a 802.11 WLAN network that shares a common set of multi-radio devices with another network, say CO-NETWORK, and we discuss WiMAX as one example of CONETWORK. One multi-radio device may not actively operate in WLAN when the same device is transmitting or receiving in the CO-NETWORK. As such, two networks interact with each other via shared multi-radio devices; and scheduling in CO-NETWORK may affect the performance of WLAN. In this paper, we study how the fairness/throughput of a WLAN network may be affected by the scheduling of CO-NETWORK. We further propose some scheduling optimization criteria for CO-NETWORK to minimize such impact. Simulation and analytical results are provided to support our discussions.
\end{abstract}

\section{Introduction}

The fast development of wireless technologies in recent years is evidenced by the proliferation of wireless standards such as IEEE 802.11, IEEE 802.16, and IEEE 802.15. As different technologies aim to provide wireless connections in different environments, it is likely that multiple technologies will coexist. Moreover, future wireless devices will likely be equipped with multiple radios of various technologies. We refer to such a device as Multi-radio Device (MRD) in this paper. Figure 1 shows several MRDs equipped with both WLAN radios and WiMAX radios.

With MRD, it is often desirable to let all radios equipped on the same device connect to their respective networks at the same time. On the other hand, there can be resource constraints among the co-located radios due to mutual interference or shared hardware component, which prevent them from operating at exactly the same time. For example, WLAN and Bluetooth radios operate at overlapping spectrum and they will interfere with each other when operating simultaneously. When WiMAX operates at $2.5 \mathrm{GHz}$ frequency band, similar problems exist between WLAN and WiMAX radios due to out-of-band emission and receiver 
saturation. If simultaneous operations of multiple co-located radios are not possible, time sharing mechanisms can be adopted such that co-located radios operate in TDM (Time-Division-Multiplex) fashion and the MRD frequently switches between different networks (at packet level) to allow perceived concurrent connections. One example of such TDM operations is the Packet Traffic Arbitration (PTA) mechanism defined in IEEE 802.15.2 [2] to resolve the mutual interference between co-located WLAN and Bluetooth radios.

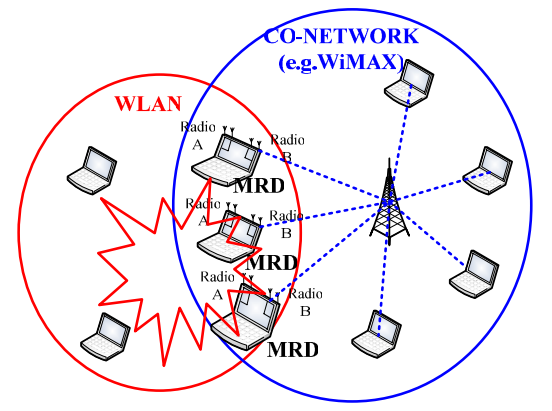

Fig. 1. Multi-radio Devices serve in both WLAN and CO-NETWORK

It is practically possible for MRD to perform TDM operations while maintaining perceived concurrent connections, since typical networks are designed such that no single radio will saturate the network. Since one device will not be busy all the time in one network (NETWORK1), this device can possibly find some time to serve in the second network (NETWOKR2). Figure 2 illustrates the interleaved activities of one MRD, where it serves in NETWORK 1 for some time and switches to NETWORK 2, then switches back to NETWORK 1 again and so on.

\begin{tabular}{|c|c|c|c|}
$\begin{array}{c}\text { TX/RX in } \\
\text { NETWORK1 }\end{array}$ & $\begin{array}{c}\text { TX/RX in } \\
\text { NETWORK2 }\end{array}$ & $\begin{array}{c}\text { TX/RX in } \\
\text { NETWORK1 }\end{array}$ & $\begin{array}{c}\text { TX/RX in } \\
\text { NETWORK2 }\end{array}$ \\
Time
\end{tabular}

Fig. 2. Interleaved activities of one MRD being active in two networks

We refer to networks that consist of MRDs as Intermittent Networks. Figure 1 illustrates an intermittent WLAN and an intermittent CO-NETWORK that share a common set of MRDs. It is necessary for MRDs to maintain concurrent connections to both networks in some usage scenarios. For example, a MRD connects to Internet via CO-NETWORK (e.g., WiMAX), while using WLAN to synchronize files with personal devices or connect multi-player gaming devices. In order to maintain concurrent connections in both WiMAX and WLAN networks, the MRD operates in TDM fashion and frequently switches between WLAN and WiMAX networks. 
As we know, 802.11 medium access control (MAC) has been designed with the assumption that radios are continuously available for use when needed. Now, given MRD radios with temporal service disruptions (to serve in CONETWORK), such assumptions no longer hold and WLAN behavior/performance may be affected. Specifically, with temporal disruptions, a MRD WLAN radio has less time to access the channel compared with a standalone WLAN radio. Consequently, the transmissions a MRD can obtain will be less and it largely depends on the disruption profile of WLAN network, which is defined as the relative occurrence time, duration and frequency of disruptions among all MRD radios of the WLAN. Additionally, a MRD WLAN radio will actively compete for the channel access only if it is not in disruption. The more MRD WLAN radios in disruption simultaneously, the fewer radios that compete for channel access in WLAN during that time. Therefore, the channel contention level of WLAN also depends on the disruption profile. Since disruption of a MRD WLAN radio occurs because the MRD needs to serve in CO-NETWORK, scheduling of CO-NETWORK directly affects disruption profile of WLAN, which in turn, affects performance of WLAN network. In this paper, we specifically study how the fairness/throughput of a WLAN network may be affected by the scheduling of CO-NETWORK. We also discuss some scheduling optimization criteria for CO-NETWORK to minimize such impact.

Throughout this paper, we consider a mixed network with both standalone WLAN radios and MRD WLAN radios. In the analysis, we assume a saturated network in which all WLAN radios have packets to transmit all the time. We also assume a MRD WLAN radio will NOT be able to sense the channel during disruptions, thus will have its backoff counter frozen. The rest of this paper is organized as follows. Related work is discussed in Section II. "Soft-fairness" in intermittent WLAN is introduced in Section III. In Section IV, we model two different disruption profiles, i.e., random disruptions and synchronized disruptions, and discuss their different impacts on achieving soft-fairness in WLAN. In Section V, we further discuss a "controlled" disruption profile and show that optimized controlled disruption profile can help reduce the impact of CO-NETWORK on WLAN. The implication of controlled disruption profile on the scheduling of CO-NETWORK, in particular, WiMAX, is also discussed in Section V. Both analytical results and simulation results are presented in Section VI to show that, optimized controlled disruption profile leads to very little impact on WLAN in terms of both throughput and fairness. Section VII finally concludes this paper.

\section{Related work}

Multi-radio networks have begun to attract attention in the past a few years. In the context of cognitive radio and spectrum sensing, there have been some recent efforts [3] [4] [5] on how to enable coordination among heterogeneous (i.e., 
using different wireless technologies) transceivers that are located on separate devices. A light-weight cooperative sensing mechanism was proposed in [3] to increase the probability that a secondary user detects a primary user to avoid interference. Reactive/proactive interference avoidance mechanisms were proposed in [4] [5] to address the co-existence of 802.11b and 802.16a networks, by adaptively adjusting transmitter PHY parameters such as frequency, power and time occupancy.

It was suggested in [1] to control protocols, power levels, antenna beam forming, frequency, coding, and timing, in order to mitigate mutual interference among multiple radios that are in close proximity or even on the same platform. The European Ambient Network Project [6] proposes a new architecture to enable the cooperation of heterogeneous networks belonging to different operator or technology domains. A generic link layer is suggested within this architecture to manage dynamic changes of radio access technologies [6]. Gao et al. [7] examined issues in building a ubiquitous QoS framework over the heterogeneous networks. Rossi et al. [8] investigated the issues related to logical device aggregation in heterogeneous networks.

In a more recent work, Zhu and Yang [9] [10] consider radios on a multiradio device as disruptive radios (due to time sharing operations). Authors studied fairness issues of WLAN networks that consist of disruptive radios and proposed an adaptive credit payback algorithm to compensate the lost transmission opportunities of disruptive radios. In this paper, we further study how different disruption profiles may affect the compensation capability, fairness as well throughput of WLAN networks. We also discuss some scheduling guideline for CO-NETWORK that can help ease the stress on WLAN. To our best knowledge, this is the first paper that addresses the scheduling issues across two different types of networks.

\section{Soft-fairness in intermittent WLAN}

Using 802.11, a standalone WLAN transmitter will transmit when the channel is sensed idle and its backoff counter reaches zero. On the other hand, for a WLAN radio located on the $\mathrm{MRD}$, it suffers transmission disruption since its channel access procedure can be interrupted by other co-located radios, and it may not be able to transmit if the radio is in disruption (even when the channel is sensed idle and its backoff counter reaches zero). As such, the channel access opportunities a MRD WLAN radio can obtain will be less compared with standalone radios, and they vary with disruption profile of the WLAN network.

For practical purpose, it is often desirable to decouple the channel access opportunities a MRD WLAN radio can obtain from the disruption profile of the WLAN. We define "soft-fairness" for a WLAN network as that, given a sufficiently long period of time, each MRD WLAN radio can obtain its desired share of transmissions (transmissions can succeed or fail), with reference to the amount of transmissions a standalone WLAN radio with the 
same channel status obtains. More specifically, if a standalone radio with the same channel status obtains $Y$ transmissions, radio $j$ shall obtain $c^{(j)} Y$ transmissions for the same period of time, where $c^{(j)}$ is a predefined parameter and $c^{(j)} \leq 1$ for MRD WLAN radios.

Without loss of generality, we consider two WLAN radios, $u$ and $v$, which share the same channel status, while radio $u$ is a standalone radio and radio $v$ is a MRD WLAN radio. When there is no disruption, the average number of backoff slots between two consecutive transmissions for stations $u$ and $v$ is denoted as $\bar{X}$. Radio $v$ will obtain fewer transmissions than radio $u$ as it suffers disruptions. However, we can allow some levels of backoff compensation for radio $v$ so that it can regain some of its lost transmission opportunities. More specifically, let $\bar{S}^{(v)}$ be the average number of extra idle slots radio $v$ spends for each transmission obtained (e.g., extra idle slots can include idle slots during disruptions). Also let $r^{(v)}$ be the backoff compensation ratio of radio $v$ and $\bar{x}^{-(v)}$ be the average number of backoff slots for station $v$. Using backoff compensation function $\bar{x}^{(v)}=\frac{1}{c^{(v)}} \bar{X}\left(1-r^{(v)}\right)$, we have derived in reference [10] the sufficient and necessary condition to achieve soft-fairness in a MIXED intermittent WLAN as follows: $r^{(v)}=c^{(v)}{ }^{-(v)} / \bar{X}$

Equation (1) gives us the optimal compensation ratio for any radio $v$. In deriving Equation (1), it has been assumed that the backoff compensation does not affect $\bar{X}$. The assumption will hold, for example, when a constant contention window size is applied. This assumption will be relaxed later in Section 6 using exponential backoff.

\section{Effect of different disruption profiles on intermittent WLAN network}

MRD WLAN radio can regain its desired fair share of channel access opportunities using backoff compensation, as we discussed in previous section. However, backoff compensation effectively reduces backoff slots of MRD WLAN radios and allows them to transmit more aggressively. If the backoff compensation ratios for MRD WLAN radios are large, the collision probability in the network can substantially increase and the overall channel utilization (i.e., the portion of channel bandwidth used for successful transmissions) will degrade. Therefore, it is good to have backoff compensation ratio as small as possible, while, at the same time, achieving desired soft-fairness. In this section, we study two different disruption profiles, i.e., random disruptions and synchronized disruptions. We analytically derive the optimal backoff compensation ratios for each disruption profile, and thus gain some insight on how much disruption 
profiles can affect the required backoff compensation ratios. The following notations are common for both disruption profiles considered here:

$N$ : the total number of WLAN radios in the network

$N_{M R D:}$ the number of MRD WLAN radios

$T_{d}$ : disruption period between two consecutive disruptions of a MRD radio

$L$ : $\quad$ length of each disruption interval, assumed to be a constant

$M_{\mathrm{d}}$ : $\quad$ average number of disruptions a MRD WLAN radio encounters between two successive transmissions

\subsection{Random Disruption Profile}

In the random disruption profile, the disruptions of each MRD WLAN radio occur randomly and independently at each slot with probability $p$. Each disruption lasts for $L$ duration. Fig. 3 shows the channel access procedure of a MRD WLAN radio. Since a MRD WLAN radio cannot sense the channel status when in disruptions, it will pause its backoff procedure by freezing its backoff counter whenever a disruption occurs. It resumes its normal backoff procedure when the disruption ends. The average duration between two consecutive disruptions of a MRD radio can be written as:

$$
E\left[T_{d}\right]=\sum_{i=1}^{\infty}(1-p)^{i-1} \times p \times(L+i-1)=\frac{1}{p}+L-1
$$

That is, the average period between two consecutive disruptions is $1 / p+L-1$, during which a MRD WLAN radio can access the channel actively for $1 / p-1$ slots and stay in disruption for $L$ slots.

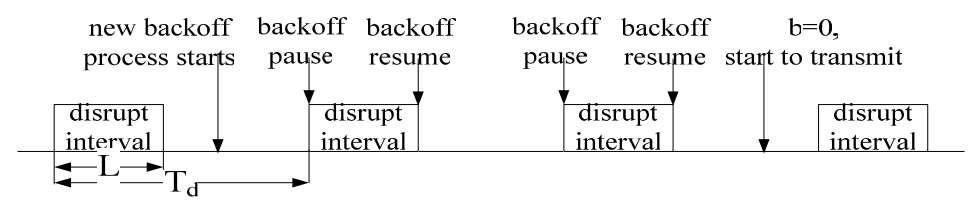

Fig. 3. Channel access procedure of MRD WLAN radios

For a MRD radio $v$ with initial backoff counter set to $\bar{X}\left(1-r^{(v)}\right) / c^{(v)}$, radio $v$ needs to go through $\bar{X}\left(1-r^{(v)}\right) / c^{(v)}$ idle slots to reduce its backoff counter to zero before transmitting. Let $\delta$ be the average probability of a slot being sensed idle so that the backoff counter may be reduced by 1 . Since disruptions of each MRD radio follow i.i.d at each slot, the number of radios in disruptions at each slot will follow the same distribution once entering steady state. As a result, $\delta$ is the same for each slot, and $\frac{1}{c^{(v)}} \bar{X}\left(1-r^{(v)}\right) \frac{1}{\delta}$ is the required average undisruptive duration (idle and busy) between two successive transmissions of radio $v$. On the other hand, since a radio always has packets to transmit, a new backoff procedure 
will be initiated immediately after the previous transmission ends. Assuming packet transmission duration $T$ is much smaller than the undisruptive duration so that edge effect may be ignored, we have

$$
\frac{1}{2}\left(\frac{1}{p}-1\right)+\left(\frac{1}{p}-1\right)\left(M_{d}-1\right)+\frac{1}{2}\left(\frac{1}{p}-1\right)=\frac{\bar{X}\left(1-r^{(v)}\right) / c^{(v)}}{\delta}
$$

The two $\frac{1}{2}\left(\frac{1}{p}-1\right)$ items at the LHS of Eq. (3) account for average undisruptive durations before the first disruption and after the last disruption respectively, while $\left(\frac{1}{p}-1\right)\left(M_{d}-1\right)$ accounts for the undisruptive duration in the middle of $M_{d}$ disruptions. From Eq. (3), we have $M_{d}=\frac{\bar{X}\left(1-r^{(v)}\right)}{\left(\frac{1}{p}-1\right) c^{(v)} \delta}$

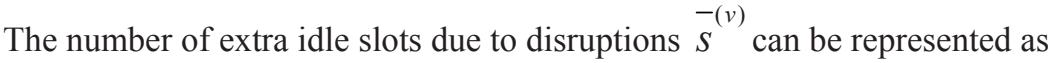
$\bar{s}^{(v)}=M_{d} L \delta$

Combine Eqs. (4) and (5) with Eq. (1), we have

$$
r^{(v)}=L /\left(L+\frac{1}{p}-1\right)
$$

Equation (6) gives the optimal backoff compensation ratio for random disruptions with disruption probability of $p$. We define disruption ratio as the percentage of time a radio in disruptions. Notice that $L /\left(L+\frac{1}{p}-1\right)$ is in fact the disruption ratio for random disruptions.

\subsection{Synchronized Disruption Profile}

Now we consider a different disruption profile, namely synchronized disruptions, where disruptions to all MRD WLAN radios happen at the same time and they all last for $L$ duration. Additionally, disruptions of each MRD radio repeat with a fixed period of $T_{d}$. As shown in Figure 4, within each disruption period $T_{d}$, the channel contention status is split into two phases: disruptive phase and undisruptive phase. In the disruptive phase, $N-N_{M R D}$ radios compete for the channel access, while all $N$ radios compete for channel access in the undisruptive phase. As such, the probability of a slot being sensed idle in disruptive phase (denoted as $\delta_{d}$ ) is quite different from that probability during undisruptive phase (denoted as $\delta_{n d}$ ).

Following similar derivation as that for random disruptions, we have the following equation for any MRD radio $v$ : 


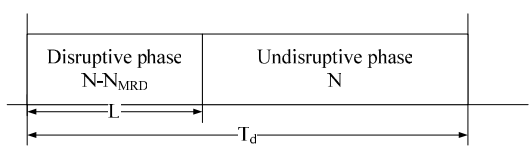

Fig. 4. Synchronized disruptions

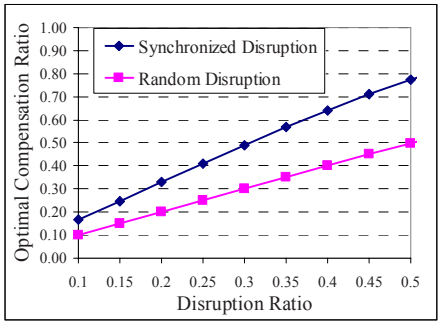

Fig. 5. Optimal backoff compensation ratio comparison

$$
\frac{T_{d}-L}{2}+\left(T_{d}-L\right)\left(M_{d}-1\right)+\frac{T_{d}-L}{2}=\frac{\frac{1}{c^{(v)}} \bar{X}\left(1-r^{(v)}\right)}{\delta_{n d}}
$$

$M_{d}$ can thus be obtained from Eq. (7), and the average number of extra idle slots due to disruptions $\bar{S}^{(v)}$ can be obtained as $\bar{S}^{(v)}=M_{d} L \delta_{d}$. As such, we have

$$
r^{(v)}=c^{(v)}{ }_{s}^{-(v)} / \bar{X}=\frac{\delta_{d} L / T_{d}}{\delta_{d} L / T_{d}+\delta_{n d}\left(1-L / T_{d}\right)}
$$

Equation (8) gives the optimal backoff compensation ratio in the case of synchronized disruptions. Notice that the channel idle time ratio during disruption phase is always higher than that of undisruptive phase (i.e., $\delta_{d}>\delta_{n d}$ ). Therefore, we observe that $r^{(v)}>L / T_{d}$, where $L / T_{d}$ is the disruption ratio in the case of synchronized disruptions.

\subsection{Discussions}

Comparing Eqs. (6) and (8), we notice that, even with the SAME disruption ratio, the optimal backoff compensation ratio for synchronized disruptions is always higher than that of random disruptions. Figure 5 shows the optimal backoff compensation ratios for random disruptions (from Eq. (6)) and synchronized disruptions (from Eq. (8)), respectively, assuming a WLAN network consisting of 15 MRD WLAN radios and 5 standalone radios. In calculating Eq. (8), a constant Contention Window size 256 is used. X-axis of Figure 5 is the disruption ratio of MRD radios; $\mathrm{y}$-axis is the optimal compensation ratio $r$. As we can see, synchronized disruptions lead to much larger compensation ratio than that of random disruptions. In particular, with disruption ratio of 0.5 , the optimal compensation ratio for random disruptions is 0.5 , while the optimal compensation ratio for synchronized disruptions is 0.78 . 


\section{Control disruptions to improve performance of intermittent WLAN network}

\subsection{Controlled Disruption Profile}

From above discussions, we have seen that disruption profiles have significant impact on the behavior of WLAN network. To achieve soft-fairness, the required backoff compensation ratio resulting from different disruption profiles can be dramatically different. Pure random disruption profile following i.i.d distribution is the ideal case, since it leads to minimal backoff compensation ratio. However, recall that a disruption at a MRD WLAN radio occurs because the MRD needs to serve in CO-NETWORK during that time. Purely random disruptions are practically impossible since disruptions among MRD WLAN radios are often correlated with each other via the scheduling of CONETWORK. As such, the question we would like to answer in this section is: what are the scheduling criteria CO-NETWORK should follow such that the required backoff compensation ratio to achieve soft-fairness in WLAN can approach the minimum.

Assuming disruptions of each MRD WLAN radio repeat with a period of $T_{d}$, we model a "controlled" disruption profile of WLAN as follows. There are $m$ disruptive intervals within each period of $T_{d}$, where $L_{d 1}, L_{d 2}, \ldots, L_{d m}$ represent the length of each disruptive interval respectively. Within the $k_{t h}(k \in[1, m])$ disruptive interval, an exclusive set of $N_{M R D_{k}}$ radios will be in disruption, which means only $N-N_{M R D_{k}}$ radios will compete for the channel access during the $k_{t h}$ disruptive interval. Note that a MRD radio will only be in disruption within one interval. Let $L_{n d}$ be the remaining undisruptive interval, during which all $N$ radios compete for the channel access. We have

$$
\sum_{i=1}^{m} L_{d i}+L_{n d}=T_{d} ; \quad \sum_{i=1}^{m} N_{M R D_{i}}=N_{M R D}
$$

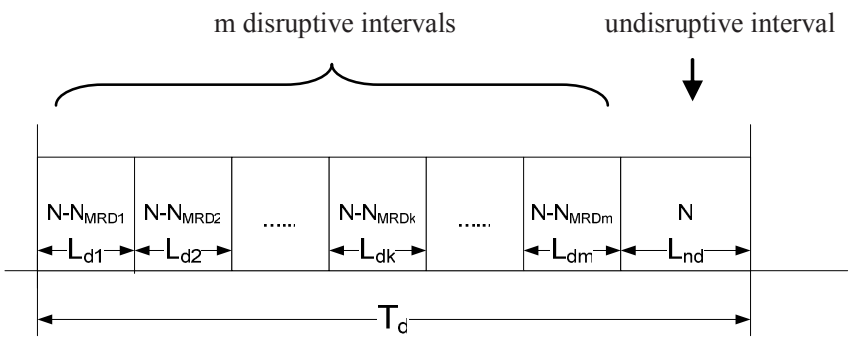

Fig. 6. Controlled Disruption Profile 
Figure 6 gives an illustration of the described controlled disruption profile. It should be noted that $m$ disruptive intervals do NOT need to be consecutive as that in Figure 6, and those intervals can appear in any order. The following derivations should not be affected.

Let $\delta_{k}$ be probability of a slot being sensed idle during the $k_{t h}$ disruptive interval and $\delta_{n d}$ be the probability of a slot being sensed idle during the undisruptive interval. We again consider a MRD WLAN radio $v$, whose disruption occurs in the $k_{t h}$ disruption interval. Using the backoff compensation function $\bar{x}^{(v)}=\frac{1}{c^{(v)}} \bar{X}\left(1-r^{(v)}\right), \bar{X}\left(1-r^{(v)}\right) / c^{(v)}$ is the number of channel idle slots radio $v$ has to go through before reducing its backoff counter to zero and transmitting. $L_{d i} \delta_{i}$ gives the number of slots being sensed idle during any $i_{t h}$ disruptive interval; and $L_{n d} \delta_{n d}$ gives the number of slots being sensed idle during the undisruptive interval. For radio $v$, we have

$$
M_{d}\left(\sum_{i=1, i \neq k}^{m}\left(L_{d i} \delta_{i}\right)+L_{n d} \delta_{n d}\right)=\bar{X}\left(1-r^{(v)}\right) / c^{(v)}
$$

The average number of disruptions, $M_{d}$, can be readily obtained from Eq. (10). The average number of extra idle slots due to disruptions for each transmission of radio $v$ can be approximated as $\bar{s}^{(v)}=M_{d} L_{d k} \delta_{k}$. Again, by applying Eq. (1), the optimal compensation ratio for radio $v$ can be obtained as:

$$
r^{(v)}=c^{(v)} \frac{\bar{s}^{(v)}}{\bar{X}}=\frac{L_{d k} \delta_{k}}{\sum_{i=1}^{m}\left(L_{d i} \delta_{i}\right)+L_{n d} \delta_{n d}}
$$

Intuitively, Eq. (11) says that the optimal backoff compensation ratio of radio $v$ equals to the number of slots sensed idle during radio $v$ 's disruption interval $L_{d k}$ divided by the total number of slots sensed idle within the period of $T_{d}$. Eq. (11) holds for any MRD WLAN radio in the network. Consider another MRD WLAN radio $\phi$, whose disruption occurs in the $q_{t h}$ disruptive interval. Based on Eq. (11), we can also write the optimal compensation ratio for $\phi$ as:

$$
r^{(\phi)}=c^{(\phi)} \frac{\bar{s}}{\bar{X}}=\frac{L_{d q} \delta_{q}}{\sum_{i=1}^{m}\left(L_{d i} \delta_{i}\right)+L_{n d} \delta_{n d}}
$$

The larger is the backoff compensation ratio, the more aggressively will a MRD radio transmit, thus the larger collision probability. To reduce the collision probability for WLAN, one way is to apply a common compensation ratio to all MRD radios and then find conditions to minimize the common compensation ratio. From Eqs. (11) and (12), it is not hard to see that, in order for 
$r^{(1)}=r^{(2)}=\ldots=r^{\left(N_{M R D}\right)}=r$, we need

$L_{d 1} \delta_{1}=L_{d 2} \delta_{2}=\ldots=L_{d m} \delta_{m}$

Combining Eqs. (11) and (13), we can obtain the common optimal

compensation ratio $r$ for all MRD WLAN radios as: $r=\frac{1}{m+L_{n d} \delta_{n d} / L_{d 1} \delta_{1}}$

Given the constraint $L_{d 1} \delta_{1}=L_{d 2} \delta_{2}=\ldots=L_{d m} \delta_{m}$, we note that, if each disruptive interval lasts for the same duration (i.e., $L_{d 1}=L_{d 2}=\ldots=L_{d m}$ ), it should be $\delta_{d 1}=\delta_{d 2}=\ldots=\delta_{d m}$. As $\delta_{d i}(i \in[1, m])$ is a function of $N_{M R D_{i}}$, Eq. (14) holds only if $N_{M R D_{1}}=N_{M R D_{2}}=\ldots=N_{M R D_{m}}=N_{M R D} / m$. That is, MRD radios should be evenly distributed into $m$ disruptive intervals. On the other hand, as we will show in Section 6 using simulation results, it is reasonably good to use $r$ given by Eq. (14) as the common compensation ratio for all MRD radios even when $\bmod \left(N_{M R D}, m\right) \neq 0$. In more general cases, if different disruptive intervals have different lengths, then the number of MRD radios in disruptions at each disruptive interval should be different. A shorter disruptive interval should have more radios in disruption, while a longer disruptive interval should have fewer radios in disruption.

Our ultimate goal is to minimize the common compensation ratio given by Eq. (14). Towards this end, we have the following two lemmas:

Lemma 1. Let $r_{l}$ be the common compensation ratio corresponding to WLAN network with $m$ disruptive intervals $L_{d 1}, L_{d 2}, \ldots, L_{d(m-1)}, L_{d m}$; and $r_{2}$ be the common compensation ratio of WLAN network with $m-1$ disruptive intervals $L_{d l}, L_{d 2}, \ldots$, $L_{d(m-l)}$. Then $r_{l}$ is always less than $r_{2}$.

Proof: In the case of $m$ disruptive intervals, let $\delta_{1}$ and $\delta_{n d}$ be the probability of a slot being sensed idle during the $1^{s t}$ disruptive interval and the undisruptive interval, respectively. The same probabilities in the case of $m-1$ disruptive intervals are represented as $\delta_{1}^{\prime}$ and $\delta_{n d}{ }^{\prime}$, respectively. $r_{1}$ and $r_{2}$ can be written as $r_{1}=\frac{1}{m+\left(T_{d}-\sum_{i=1}^{m} L_{d i}\right) \delta_{n d} / L_{d 1} \delta_{1}}, r_{2}=\frac{1}{m-1+\left(T_{d}-\sum_{i=1}^{m-1} L_{d i}\right) \delta_{n d}^{\prime} / L_{d 1} \delta_{1}^{\prime}}$

With $m$ intervals, the number of MRD radios in disruption within each disruptive interval is no greater than that with $m-1$ intervals. Lemma 1 can be proved by noting $\delta_{n d} / \delta_{1} \geq \delta_{n d}^{\prime} / \delta_{1}^{\prime} \quad$ and $\quad \delta_{n d} / \delta_{1}<1$. [end of proof]

Lemma 2. Assume the number of disruptive intervals $m$ in the WLAN network is fixed, and the number of MRD radios in disruption within each disruptive interval (i.e., $N_{M R D_{1}}, N_{M R D_{2}}, \ldots$ ) is given. Let $r_{l}$ be the common compensation 
ratio corresponding to WLAN network with $m$ disruptive intervals $L_{d 1}, L_{d 2}, \ldots$, $L_{d m}$; and $r_{2}$ be the common compensation ratio of WLAN network with $m$ disruptive intervals $L_{d 1}^{\prime}, L_{d 2}^{\prime}, \ldots, L_{d m}^{\prime}$. If $L_{d i}^{\prime} \geq L_{d i}(i \in[1, m])$, then $r_{l}$ is always less than or equal to $r_{2}$.

Proof: Let $\delta_{1}$ and $\delta_{n d}$ be the probability of a slot being sensed idle during the $1^{s t}$ disruptive interval and the undisruptive interval, respectively. $f(r)=\delta_{n d} / \delta_{1}$ is a function of $r$. Now consider two curves, $Y_{1}(r)=m+C_{1} f(r)$ and $Y_{2}(r)=m+C_{2} f(r)$, where $C_{1}=\left(T_{d}-\sum_{i=1}^{m} L_{d i}\right) / L_{d 1}>=C_{2}=\left(T_{d}-\sum_{i=1}^{m} L_{d i}{ }^{\prime}\right) / L_{d 1}^{\prime}$

$r_{1}$ and $r_{2}$ correspond to the points where $Y_{1}(r)$ and $Y_{2}(r)$ intersect with the curve $Z(r)=1 / r$, respectively. Lemma 2 can be proved by noting both $r_{1}$ and $r_{2}$ are no larger than $1 / m$. Additionally, $Y_{2}(r) \leq Y_{1}(r)<Z(r)$ when $r \rightarrow 0$ and $Z(r)<Y_{2}(r) \leq Y_{1}(r)$ when $r=1 / m$.

[end of proof]

From Lemma 1 and 2, we have the following theorem:

Theorem 1: Given that constraint in Eq. (13) is satisfied, the common optimal compensation ratio for MRD WLAN radios will decrease when the number of disruptive intervals (i.e., $m$ ) increases and the length of each disruptive interval (i.e., $L_{d 1}, L_{d 2}, L_{d 3}, \ldots, L_{d m}$ ) decreases. From theorem 1, we know that synchronized disruption profile discussed in Section 4 has the worst performance. Notice that when $L_{d 1}=L_{d 2}=\ldots=L_{d m}=L$ and $m L=T_{d}$, the optimal compensation ratio of the controlled disruption profile from Eq. (14) exactly equals to the disruption ratio $L / T_{d}$. Later in Section VI, we will use numerical results to show that, when $m$ is maximized, the optimal compensation ratio resulting from controlled disruption profile stays very close to the disruption ratio in general.

\subsection{System Implication}

As we discussed before, different disruption profiles of WLAN map to different scheduling choices for the CO-NETWORK. Consider IEEE 802.16 (WiMAX) based on Orthogonal Frequency Division Multiplexing Access (OFDMA) and Time Division Duplex (TDD) operations as an example of CO-NETWORK. Two dimensions of channel resources can be allocated to each WiMAX radio using OFDMA: one is frequency (vertical axis of Figure 7) and the other is time (horizontal axis of Figure 7). Given five MRD and five standalone (STD) WiMAX devices, Figure 7 illustrates two scheduling options for downlink (DL) operations. In the upper subfigure (Figure 7.a), five MRD WiMAX radios' DL allocations are distributed over the time domain and they are not overlapping with each other in time. Each allocation for a MRD in WiMAX network corresponds to 
a disruption of this particular device in WLAN network. Therefore, the scheduling in Figure 7.a maps to the case that there are five disruptive intervals (i.e., $m=5$ ) in the WLAN network. Another scheduling example for the WiMAX network is shown in Figure 7.b, in which DL-bursts of all five MRDs are allocated within the same time period (occupying different frequencies). Scheduling in Figure 7.b corresponds to the case that WLAN network has synchronized disruption profile.

Our discussions in this section essentially state that, if WiMAX base station follows the scheduling in Figure 7.a rather than that in Figure 7.b, the co-located WLAN network will need less backoff compensation in order to maintain the desired fairness for MRD WLAN radios. Consequently, the co-located WLAN network will suffers less throughput degradation. Theorem 1 provides guidelines for WiMAX base station to schedule MRD WiMAX radios:

a). Allocate a MRD along frequency dimension first, such that its time domain occupancy is as small as possible;

b). Evenly distribute the scheduling of MRDs into $m$ non-overlapping time intervals with equal length;

c). The number of non-overlapping time intervals $m$ should be maximized.

The above guidelines can be applied to scheduling within one frame as well as scheduling across multiple frames. How to design specific scheduling algorithm that follows the above guidelines for WiMAX networks consisting of MRDs without hurting WiMAX performance is an on-going work.

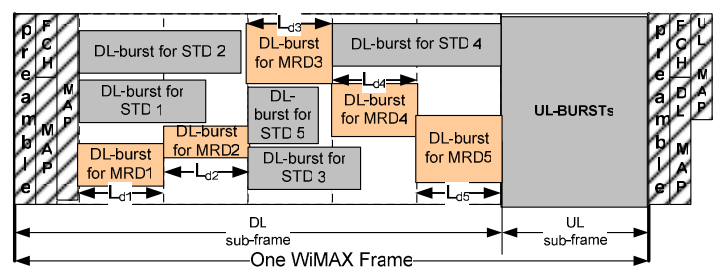

(a) Recommended Scheduling

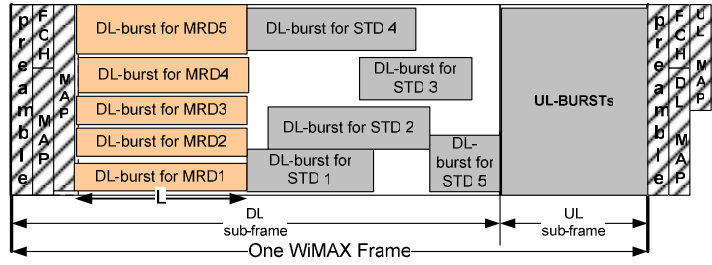

(b) Worse Scheduling

Fig. 7. Scheduling Choices for Co-located WiMAX Network 


\section{Numerical Results and discussions}

\subsection{Simulation Model}

We simulate the disrupted channel access behavior of WLAN network using code written in MATLAB ${ }^{\mathrm{TM}}$, where time is sliced into slots. A slot is considered as Success if only one radio is transmitting; considered as Collision if more than one radios are transmitting; and considered as Idle if no radio is transmitting. We concentrate on "Saturate Performance", where all WLAN radios always have packets to transmit. To validate our simulator, we have simulated the WLAN network with standalone WLAN radios only, and compared the throughput against the theoretical results from reference [11]. Fig. 8 shows that simulation results match very well with theoretical results from reference [11]. Data points from simulations are obtained with running time sufficiently long to reach steady state. There are two groups of radios in the WLAN network: standalone WLAN radios and MRD WLAN radios. In the simulations, radios in the same group share the same configuration. Fairness Index is calculated as the number of transmissions averaged over all MRD WLAN radios divided by the number of transmissions averaged over all standalone WLAN radios. Throughout our simulations, the desired share factor $c$ for all MRD radios is set to 1 . Therefore, if the measured fairness index equals to 1 , then WLAN achieves perfect softfairness.

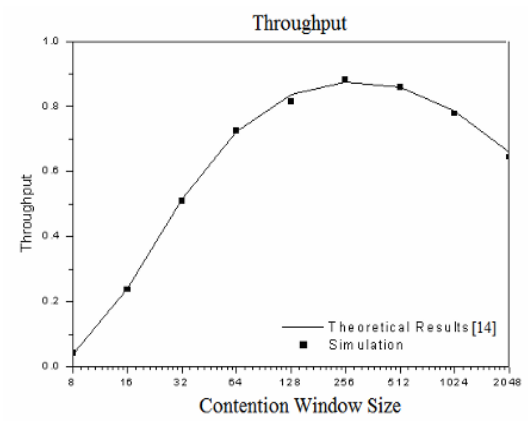

Fig. 8. Throughput of WLAN network with 20 standalone radios

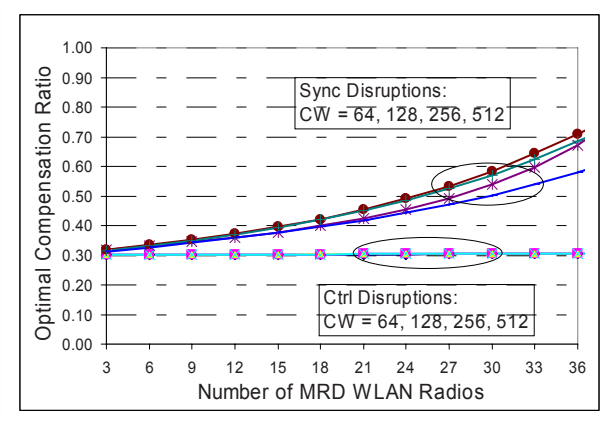

Fig. 9. Optimal Compensation Ratios from Analytical Model

\subsection{Throughput and fairness of WLAN with varying number of MRD radios}

We consider a mixed WLAN network with total 40 radios. The number of MRD WLAN radio (denoted as $N_{M R D}$ ) is increased from 3 to 36 with a step size of 3; the number of standalone radios decreases accordingly. Disruption period $T_{d}$ is set to 
1000 slots; each MRD WLAN radio will be in disruption for 300 slots during each disruption period $T_{d}$ (i.e., disruption ratio of 0.3 for each MRD radio). Packet transmission duration $T$ is set to 10 slots. Disruptions of all MRD radios occur at the same time in the case of synchronized disruption profile. For controlled disruption profile, MRD radios are evenly distributed into 3 disruptive intervals; there are $N_{\text {MRD }} / 3$ MRD radios in disruption within each disruptive interval; the remaining undisruptive interval lasts for 100 slots. Figure 9 shows the optimal compensation ratios derived from our analytical models for both synchronized disruption profile and controlled disruption profile, using various constant contention window sizes. In the figure, we marked curves for synchronous profile as "Sync", while the curves for controlled profile as "Ctrl". X-axis is the number of MRD radios while y-axis is the optimal compensation ratio. As we can see, with synchronized profile, the optimal compensation ratio is very sensitive to the contention window size $(\mathrm{CW})$; different $\mathrm{CW}$ size leads to different optimal compensation ratio. Additionally, the optimal compensation ratios for synchronized disruption profile increase rather rapidly when the number of MRD radios increases; the value reaches 0.7 with $36 \mathrm{MRD}$ radios, which is much larger than the disruption ratio (i.e., 0.3). On the other hand, the optimal compensation ratios resulting from controlled disruption profile are not sensitive to contention window sizes; four curves corresponding to four different $\mathrm{CW}$ sizes override with each other. Furthermore, the optimal compensation ratios for controlled profile remain close to the disruption ratio 0.3 despite the increase of MRD radios.

Figure 10 shows the fairness index obtained from simulations, when applying the optimal compensation ratios in Figure 9 to the corresponding scenarios. Xaxis is the number of MRD radios; $y$-axis is the measured fairness index. Figure 10 serves the purpose of validating our analytical models. As we can see, for both synchronized disruption profile and controlled disruption profile, the fairness index obtained from simulations stays around 1 in all simulated scenarios, which confirms that our analytical models are reasonably accurate in modeling the optimal compensation ratio.

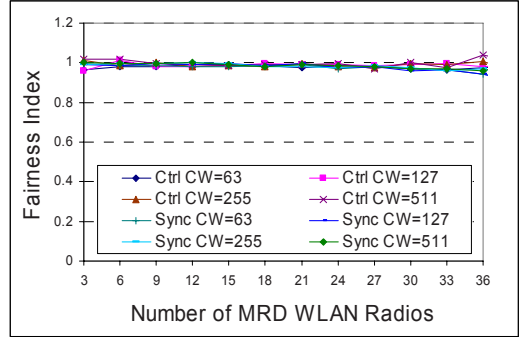

Fig. 10. Fairness Index from Simulations

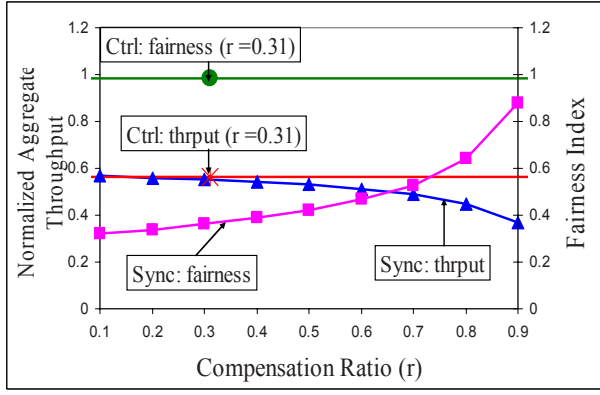

Fig. 11. Throughput and Fairness of WLAN using Exponential Backoff 
We further proceed to enable the exponential backoff for all WLAN radios. That is, whenever a transmission fails, the sender will double its CW size until the maximum $\mathrm{CW}\left(\mathrm{CW}_{\max }\right)$ is reached. We use $\mathrm{CW}_{\min }=31$ and $\mathrm{CW}_{\max }=511$ in the simulations. We simulated a WLAN network consisting of $33 \mathrm{MRD}$ radios and 7 standalone radios. From Figure 9, we have seen that, for synchronized disruption profile, there is no single compensation ratio that works for all sizes of $\mathrm{CW}$. Therefore, we applied compensation ratio from 0.1 to 0.9 (x-axis) for all MRD radios in the case of synchronized profile, and plotted both normalized aggregate throughput (left y-axis) and fairness (right y-axis) of WLAN in Figure 11. As we can see, when compensation ratio $\mathrm{r}$ is increased from 0.1 to 0.9 , fairness index improves from 0.32 to 0.88 , but aggregate throughput decreases from 0.57 to 0.37 for synchronized profile. In other words, either fairness or throughput will suffer for synchronized disruption profile. On the other hand, for controlled disruption profile, the optimal compensation ratio is insensitive to $\mathrm{CW}$. When we apply $\mathrm{r}=0.31$ to the same WLAN network with controlled disruption profile, we achieved throughput of 0.56 (peak value of synchronized profile) AND fairness index of 0.99 at the same time, as two flat lines in Figure 11 show. That is, controlled disruption profile allows WLAN to maintain good performance in both fairness and throughput.

\subsection{Throughput and fairness of WLAN with varying disruption ratios}

In this subsection, we study the performance of controlled disruption profile for various disruption ratios. We fix the number of MRD radios as 15 (i.e., $N_{M R D}=$ 15 ) and the number of standalone radios as 5. Disruption ratio is increased from 0.1 to 0.5 with a step size of 0.05 . The same disruption ratio applies to all MRD radios. For controlled disruption profile, the number of disruptive intervals (i.e., $m$ ) should be maximized, and it depends on the value of disruption ratio. For example, if disruption ratio is 0.4 , there can only be two non-overlapping disruptive intervals. With $15 \mathrm{MRD}$ radios, one interval will have $8 \mathrm{MRD}$ radios in disruption and the other interval will have $7 \mathrm{MRD}$ radios in disruption. We calculate the optimal compensation ratio based on Eq. (14) using $N_{M R D_{1}}=\left\lceil N_{M R D} / m\right\rceil$, the values are shown in Figure 12 for different constant $\mathrm{CW}$ sizes. X-axis of Figure 12 is the disruption ratio; $\mathrm{y}$-axis is the optimal compensation ratio. The disruption ratio in each case is also plotted as a curve for ease of reading. Again, as we can see, the optimal compensation ratio for controlled profile is quite insensitive to $\mathrm{CW}$ sizes. The optimal compensation ratio can become slightly larger than the disruption ratio when the undisruptive interval becomes relatively large (e.g., when $L_{n d}=0.3 T_{d}$ with disruption ratio of 0.35 ); but it stays close to the disruption ratio in general. Comparing Figures 5 and 12, it is 
also not hard to see that synchronized disruption profile require much larger compensation ratios than controlled disruption profile.

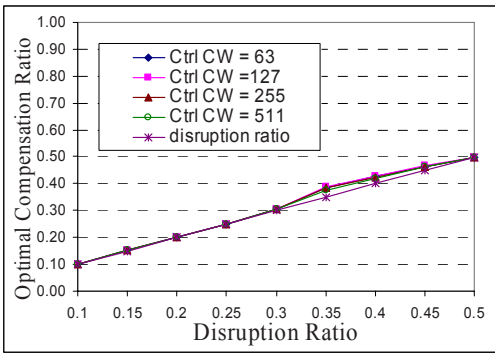

Fig. 12. Optimal Compensation Ratios for Controlled Disruptions

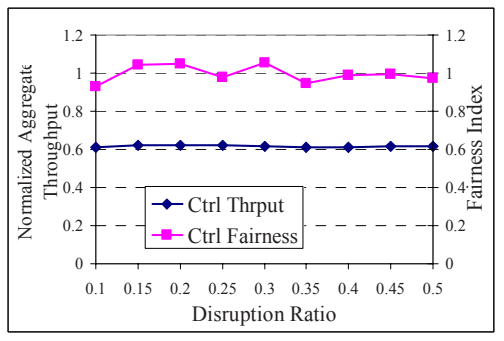

Fig. 13. Throughput and Fairness using Exponential Backoff

The optimal compensation ratio for each disruption radio is average over different $\mathrm{CW}$ sizes, and is then applied to all MRD radios in the simulations. Exponential backoff of WLAN is enabled in the simulations. Figure 13 shows the normalized aggregate through (left y-axis) and fairness index (right y-axis) of WLAN at various disruption ratios (x-axis). As we can see, even though it is not possible to evenly distribute MRD radios into $m$ disruption intervals in some cases ( since $\left.\bmod \left(N_{M R D}, m\right) \neq 0\right)$, the compensation ratios calculated from Eq. (14) are reasonably good and fairness index in all simulated scenarios stay close to 1 . At the same time, WLAN aggregate throughput is hardly affected by the change of disruption ratios when controlled disruption profile is applied.

\section{Conclusions}

In this paper, we consider a WLAN network using 802.11 that shares a common set of multi-radio devices with another network CO-NETWORK (e.g., WiMAX). One multi-radio device may not actively operate in WLAN when the same device is transmitting or receiving in the CO-NETWORK. As such, two networks interact with each other via shared multi-radio devices; and scheduling in CONETWORK may affect the performance of WLAN. We studied how fairness/throughput of a WLAN network may be affected by different disruption profiles. We show via analytical models and simulation results that, disruptions will have less impact on WLAN performance if disruptions follow the optimized controlled disruption profile discussed in this paper. Our results provide scheduling guideline for the CO-NETWORK to alleviate its impact on WLAN performance. As an example of CO-NETWORK, scheduling in WiMAX network is briefly discussed. 


\section{References}

1. J. Lansford, "UWB Coexistence and Cognitive Radio", Ultra Wideband Systems, May 18-21, 2004.

2. IEEE Std 802.15.2-2003, "Coexistence of Wireless Personal Area Networks with Other Wireless Devices Operating in Unlicensed Frequency Bands".

3. S. M. Mishra, A. Sahai, and R. W. Brodersen, Cooperative Sensing among Cognitive Radios, IEEE ICC 2006.

4. X. Jing, S.-C. Mau, D. Raychaudhuri and R. Matyas, "Reactive Cognitive Radio Algorithms for Co-Existence between IEEE 802.11b and 802.16a Networks", Proceedings of IEEE Globecom, St. Louis, MO, Nov. 28-Dec. 2, 2005.

5. X. Jing and D. Raychaudhuri, "Spectrum Co-existence of IEEE 802.11b and 802.16a Networks using the CSCC Etiquette Protocol", Proceedings of IEEE DySPAN (International Symposium on New Frontiers in Dynamic Spectrum Access Networks), Baltimore, MD, Nov. 8-11, 2005.

6. N. Niebert, A. Schieder, H. Abramowicz, G. Malmgren, J. Sachs, U. Horn, C.Prehofer, and H. Karl, "Ambient networks: An architecture for communication networks beyond 3g," IEEE Wireless Communications, vol. 11, pp. 14--22, April 2004.

7. X. Gao, G. Wu, and T. Miki, "End-to-end qos provisioning in mobile heterogenous networks," IEEE Wireless Communications, vol. 11, pp. 24--34, June 2004.

8. M. Rossi, L. Badia, P. Giacon, and M. Zorzi, "On the effectiveness of logical device aggregation in multi-radio multi-hop networks," in Proceedings of the 3rd IEEE International Workshop on Mobility Management and Wireless Access (MobiWac 2005), Maui, Hawaii, USA, 2005.

9. J. Zhu, X. Yang and X. Guo, "Disruptive CSMA with Credit Payback (CP) Protocols for MultiRadio Network," CrownCom 2007, August 2007.

10. X. Yang, J. Zhu and X. Guo, "Using "Scaled Credit Payback" to Achieve Soft-fairness for Disruptive Radios in CSMA Networks", IEEE SECON 2007 poster session.

11. G. Bianchi, Performance Analysis of IEEE 802.11 Distributed Coordination Function, IEEE JSAC, vol. 8, no.3, March 2000. 